\title{
Thrombosis, an Important Piece in the COVID-19 Puzzle - From Pathophysiology to Therapy
}

\author{
Elena Bobescu ${ }^{1,3}$, Lorena Dima ${ }^{2}$, Luigi Geo Marceanu ${ }^{1 *}$, Marius Alexandru Moga ${ }^{1,4}$, Liliana \\ Marcela Rogozea ${ }^{2}$, Alexandru Covaciu ${ }^{1,3 *}$ and Larisa Alexandra Vladau ${ }^{1,3}$ \\ ${ }^{1}$ Department of Medical and Surgical Specialties, Transilvania University of Brasov, Romania
}

${ }^{2}$ Department of Fundamental, Prophylactic and Clinical Disciplines, Transilvania University of Brasov, Romania

${ }^{3}$ Department of Cardiology, Clinical County Emergency Hospital Brasov, Romania

${ }^{4}$ Clinical Hospital of Obstetrics and Gynecology "Dr. I.A. Sbarcea", Romania

*Corresponding author: Luigi Geo Marceanu, Transilvania University of Brasov, Faculty of Medicine, Department of Medical and Surgical Specialties, Brasov, Romania. Alexandru Covaciu, Transilvania University of Brasov, Faculty of Medicine, Department of Medical and Surgical Specialties, Brasov, Clinical County Emergency Hospital Brasov, Department of Cardiology, Brasov, Romania.

To Cite This Article: Luigi Geo Marceanu, Alexandru Covaciu, Thrombosis, an Important Piece in the COVID-19 Puzzle - From Pathophysiology to Therapy. Am J Biomed Sci \& Res. 2021 - 11(4). AJBSR.MS.ID.001648. DOI: 10.34297/AJBSR.2021.11.001648.

Received: 阱 December 21, 2020; Published: 眥 January 12, 2021

\begin{abstract}
Background: A lot of data about COVID-19 was already published but this is only a part of the pandemic puzzle pieces. Once we will have all the pieces together, we will be able to successfully treat this disease with so many challenges. This infectious disease has a high thrombogenic potential. The study aims to review published data about COVID-19 associated thrombosis, from pathophysiology to treatment and the role in patient's evolution. Data sources: We have searched for articles and studies published in online.

Platforms: MEDLINE/PubMed database, Google Scholar, ScienceDirect, Wiley Online Library, Nature Public Health Emergency Collection. Results: We have found numerous articles regarding the COVID-19 infection. We selected only those focused on the thrombosis. D-dimers had predictive value in identifying thrombosis and high level had correlated with the severity of the infection and death. The most patients who were on chronic anticoagulant therapy before contacting the virus had a better prognosis. Heparin has other favorable effects such as a direct antiviral and anti-inflammatory effect in addition to its anticoagulant effect.

Conclusions: COVID-19 infections are frequently complicated by the thrombotic pathology. High plasma level of D-dimers is predictive factor for severe prognosis and the recommended anticoagulant therapy associated with low mortality is heparin followed by direct oral anticoagulants. Randomized studies in large groups of patients and therapeutic guidelines are still needed.

Keywords: COVID-19; Thrombosis Pathophysiology; D-dimers; Anticoagulants Therapy

Abbreviations: COVID 19: Corona Virus Disease 2019; D-dimers: Fibrin degradation products; PRISMA: Preferred Reporting Items for Systematic Reviews and Meta Analysis; VTE: Venous thromboembolism; DIC: Disseminated Intravascular Coagulopathy; UFH: Unfractionated Heparin; LMWH: Low molecular weight heparin ACE2: Angiotensin-converting enzyme 2 receptor; 02: Oxygen for Peer Review; DOACs: Direct oral anticoagulants; VKA = Vitamin K antagonists; SARS-COV-2 = Severe acute respiratory syndrome coronavirus 2; INR = International normalized ratio of prothrombin time; TeleCheck-AF = A communication system with three components: (1) a structured teleconsultation ('Tele'); (2) an app-based on-demand heart rate and rhythm monitoring infrastructure ('Check'); and (3) comprehensive AF management ('AF').
\end{abstract}

\section{Introduction}

The COVID 19 infection has spread across the globe in an exponential manner, quickly being declared a pandemic and causing the onset of a public health crisis and not only. More than ever, monitoring and diagnosing the patients with this disease involves multidisciplinary teams. Tele-diagnosis and tele-monitoring

became the only way to follow-up of quarantined patients in order to have information about patient status and recovery progress. Each patient will have a personalized diagnosis and treatment in correlation with acute infectious disease and history of associated diseases. For this reason, is important to integrate as soon as 
possible in current medical practice a wide spectrum of published information and the results of the studies issued so far.

In patients with COVID-19 infection it could be observed a high diversity of clinical signs and symptoms, ranging from asymptomatic patients to interstitial pneumonia and progression to acute respiratory distress syndrome, accompanied by multiple system and organ failure [1,2]. Although pulmonary pathology is more often associated, the cardiac injury is an important piece of the puzzle, frequently accompanied by thrombotic vascular complications. One of the biggest challenges facing the medical staff caring for COVID 19 patients is the prevention and early diagnosis of vascular thromboembolism.

Due to the fact this infection is a recent condition, clinicians are currently facing a lack of systemized information related to pathophysiology of the virus and complications of this infection. For this reason, we selected the publications about thromboembolic complications of COVID-19 patients, correlated and tried to make connections between all of this data. Although a remarkable progress has been made since the onset of the pandemic, randomized studies in large groups of patients and the development of therapeutic guidelines are still needed.

\section{Materials and Methods}

We have reviewed more than 190 abstracts, articles and studies published in online platforms such as MEDLINE/PubMed database,
Google Scholar, ScienceDirect, Wiley Online Library and Nature Public Health Emergency Collection. We selected only 52 articles that provided useful information in the daily medical practice, studies that analyzed the thrombus formation pathophysiology, risk factors for an unfavorable course of COVID-19 infection and we discussed the stratification of the risk of pulmonary thromboembolism, the cardiovascular complications, the importance of D-dimers and the effects of anticoagulant therapy in these patients. Also, the remote monitoring strategies of patients with a chronic need for oral anticoagulant treatment were noted. There was a difficulty in maintaining the therapeutic value of INR in quarantined patients.

\section{Results and Discussion}

As described in Figure 1, a total of 2784 related abstracts, articles and studies were identified from the database. Among these, we removed 721 repetitive publications. Of these, 531 records were excluded after the title or the abstract review because they did not refer to the complications of the disease that we focus on. Furthermore, 142 articles were excluded, because the main discussion different from our aims, they were not applicable in daily medical practice, there were clinical cases or series of clinical cases, studies whit fewer then 10 patients and also we found some articles with poor English translation. The remaining 50 studies and articles were closely evaluated and were cited in the present review.

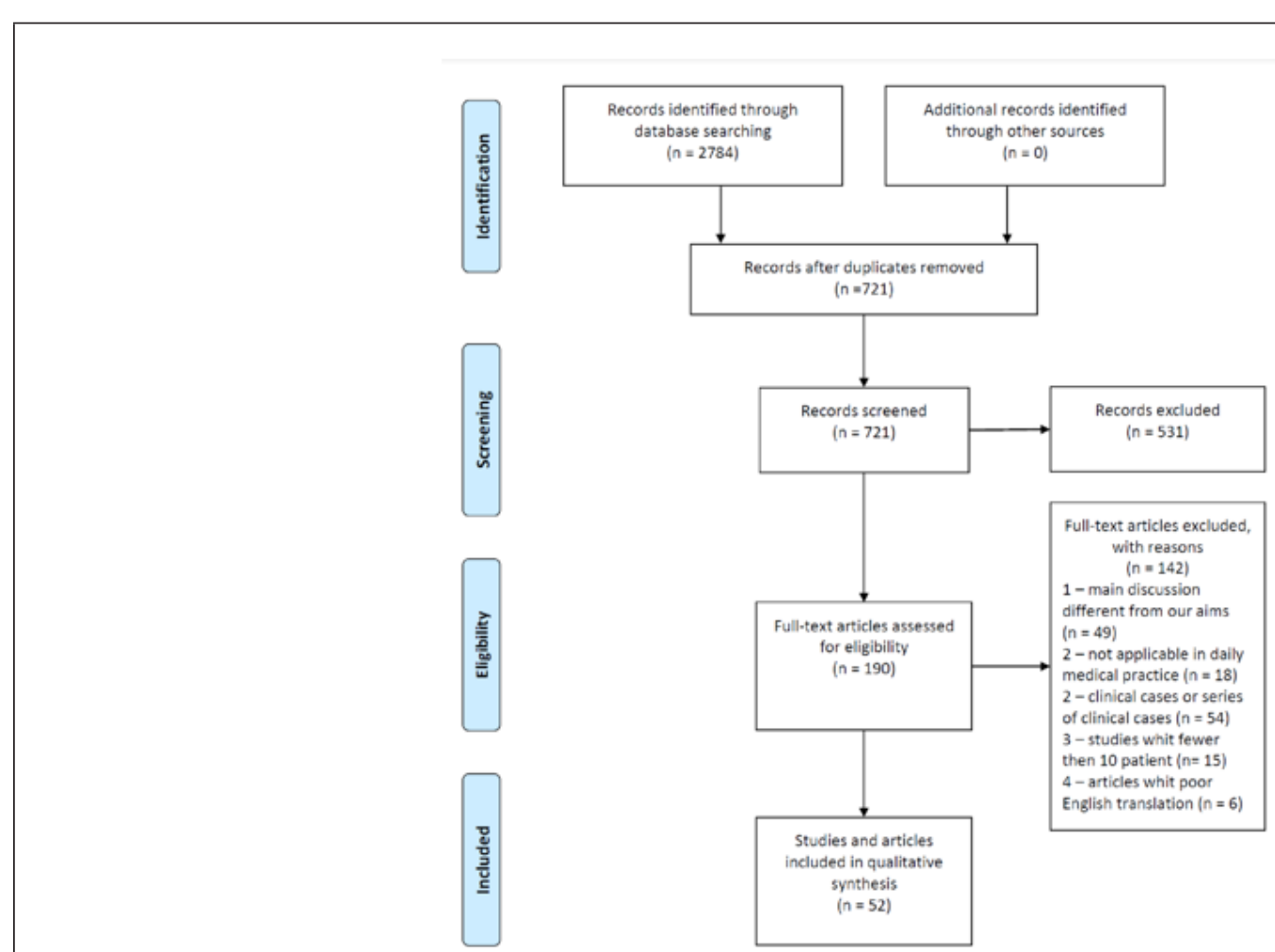

Figure 1: The flow diagram shows the selection process according to the PRISMA statement. 


\section{Elements of pathophysiology in the COVID-19 infection associated thrombosis}

The Virchow triad is a fundamental concept of interaction between three components to create a favorable environment for thrombogenesis. All these components are also found in the COVID-19 infection and they consist of blood flow abnormalities, vascular wall abnormalities and the presence of circulating procoagulant compounds [3]. Blood flow is altered by venous stasis caused by the prolonged bed rest [4]. In the case of the COVID-19 associated coagulopathy, there is a vascular inflammation and a large endothelial dysfunction, followed by arterial, venous or microcirculation thrombosis. Another feature of the COVID-19 coagulopathy is the significant increase in fibrin degradation products such as the D-dimers, followed by platelet activation and aggregation and thrombin generation which leads to the recruitment of the circulating platelets and the formation of the thrombus [3]. Thus the high platelet reactivity has an important contribution in the occurrence of COVID-19 coagulopathy [5]. In the acute coronary syndromes the endothelial dysfunction, high platelet reactivity and oxidative stress are significantly correlated [6,7], while in COVID-19 patients, the endothelial inflammation is the trigger factor of all this factors exacerbation $[8,9]$. Also, the viral infection triggers an immune and non-immune cellular response that creates an imbalance between pro and anticoagulant factors - another main mechanism of thrombus formation in COVID-19 infection [3].

Due to the inflammation from viral infection, the vascular endothelium suffers injuries, von Willebrand factor concentration increase, Toll-like receptors and the tissue factor are activated, leading to the onset of the coagulation cascade with the formation of fibrin clot [10]. The inflammation can determine the expression of tissue factor at the level of the atrial endothelium too, thus stimulating the formation of intra-atrial thrombi [11,12]. The physiological response to the excessive activity of coagulation cascade determines disruption of fibrin clot, leading to release of D-dimers [9]. In COVID-19 patients, the level of D-dimers has a good predictive value in identifying arterial or venous thrombosis $[13,14]$, also correlates with severity of the disease, development of acute respiratory distress syndrome and mortality [14]. According to Al-Ani et al. the D-dimers have a dynamic evolution, their level increasing with the progression of the disease, significantly correlated with severe prognosis and mortality $[2,15]$.

In COVID-19 coagulopathy, plasma levels of fibrinogen and platelet count are in the most cases normal or elevated, differing from the disseminated intravascular coagulation characterized by low plasma fibrinogen levels and thrombocytopenia. This explains the lower incidence of bleeding in patients treated with anticoagulants in the COVID-19 coagulopathy. The occurrence of thrombocytopenia in a patient with COVID-19 is a strong prognostic factor of the evolution towards multiple organ failure and disseminated intravascular coagulopathy (DIC). In COVID-19 severe evolution with DIC seems to be the result of cumulative factors induced sepsis and prothrombotic status $[3,16]$. Last but not least, in COVID-19 infection, the platelets, in addition to their pro-thrombotic action mediated by classical platelet receptors, also have a platelet receptor-mediated hemostatic effect with an important role in the endothelial barrier protection and microvascular integrity [17].

\section{Risk factors and complications associated with the negative evolution of COVID-19 infected patients}

Patients with cardiac pathology or cardiovascular risk factors (such as old age, diabetes, neoplastic disease) are susceptible to an unfavorable evolution towards COVID-19 infection. At the same time, the new coronavirus infection itself can lead to many cardiovascular diseases [18-20]. Inciardi et al. conducted the first clinical study that described the characteristics and evolution of patients with a personal history of cardiac pathology and COVID-19 pneumonia. The study was conducted on the population of northern Italy and it concluded that patients with heart disease hospitalized for COVID-19 had a poorer prognosis when compared to patients without a personal history of cardiac pathology. They had a higher rate of mortality, thromboembolic events and septic shock (36\% vs. $15 \%$ mortality and $57 \%$ vs. $21 \%$ major complications overall) [21]. It is considered that the cardiovascular system and the respiratory system have an equal role to in the pathogenesis of COVID-19 infection [22]. Although most suffer respiratory symptoms, a considerable number of patients develop various cardiovascular complications. In a study conducted by Gavioli et al. it has been observed that approximately $20 \%$ of hospitalized infected patients suffered cardiac injury defined by an increase in cardiac biomarkers above the 99th-percentile upper reference limit [18] with the appearance of acute coronary syndromes as a complication of the COVID-19 infection [3]. The most common complications are: arrhythmia, arterial and venous thromboembolism, cerebrovascular injury, fulminant myocarditis and heart failure that can evolve to cardiogenic shock [19,23-25]. One report of the Italian National Institute of Health showed that a quarter of the patients who died of COVID-19 had atrial fibrillation, ranking it as the fourth most common pathology associated with these patients. The New York State Department of Health reported that atrial fibrillation ranked as the 7th most frequent comorbidity among the COVID-19 patients [26]. Holt et al. conducted an analysis of medical records in Denmark by comparing the number of newly diagnosed cases with atrial fibrillation during the first three months of 2019 and 2020. There was a $47 \%$ decrease of newly diagnosed cases with atrial fibrillation during the quarantine period, suggesting a concomitant 
increase in undiagnosed patients. In the case of these patients, the lack of treatment can lead to complications of atrial fibrillation the most feared being stroke, favored by the prothrombotic status in COVID 19 patients [27].

One of the mechanisms involved in the occurrence of arrhythmias in COVID-19 patients is hypoxia, which causes anaerobic glycolysis, resulting in reduced intracellular $\mathrm{pH}$ and increased cytosolic calcium levels. This favors an early or delayed depolarization of the myocyte and it can also cause a transient alteration of the duration of the action potential. In addition, hypoxia also increases the level of extracellular potassium, which lowers the depolarization threshold and thus the electrical conduction is accelerated [28]. Another trigger for arrhythmias is the systemic inflammatory process. This favors the formation of myocardial tissue fibrosis and consequently the electrical properties of the atrial myocardium are altered $[11,29]$.

\section{The screening and diagnosis of thromboembolism in COVID-19 infected patients}

In the case of COVID-19 infected patients, one of the main challenges faced by physicians is the prevention and early diagnosis of venous thromboembolism (VTE), given the fact that there is still no standardized protocol to administrate anticoagulant treatment to these patients [20]. Llitjos et al. suggest performing a systematic screening for VTE and an early initiation of anticoagulation at therapeutic doses in patients with severe COVID-19 infection due to its increased thrombogenicity [29,30]. In the study made by Llitjos et al. it was implemented a systematic screening strategy using complete duplex ultrasound of the COVID-19 infected patients treated in intensive care units between the first and 3rd day after admission. The patients who were not diagnosed with VTE within the first screening had the duplex ultrasonography repeated on the 7 th day. All patients received the anticoagulant treatment with unfractionated heparin (UFH) or low molecular weight heparin (LMWH) in prophylactic or therapeutic doses, depending on the risk of thrombosis and bleeding associated with each patient The duplex ultrasonography was performed according to current guidelines, assessing the venous circulation from the thigh to the ankles. Pulmonary thromboembolism was systematically sought in patients with persistent respiratory failure; they were evaluated by pulmonary arteriography, by computed tomography or by transesophageal echocardiography in the case of immovable patients. The study reported an incidence of peripheral VTE of 69\%, and of pulmonary VTE of $23 \%$. The proportion of patients with VTE was significantly higher in the group treated with prophylactic doses of anticoagulant than in the group treated with therapeutic doses (100\% vs. $56 \%)$ [30].

In a study conducted in 24 hospitals in France, in which 2878 patients with COVID-19 infection were hospitalized consecutively,
1240 patients were admitted after the exclusion from the study of those who did not undergo pulmonary angiography by computed tomography to prove pulmonary thromboembolism and those admitted directly to the intensive care unit. Of the 1240 patients, 8.3\% were diagnosed with pulmonary thromboembolism and for $77.7 \%$ of them the diagnosis was confirmed within the first 48 hours of hospitalization [1]. Due to the fact that the incidence of VTE is significant higher in COVID-19 infection, venous embolism screening should be considered both in patients in a very severe clinical condition with a need for admission into intensive care units and for those with a less severe clinical condition. However, such procedures are difficult to implement because these patients are isolated [15].

\section{The effects of anticoagulants in COVID-19 infected patients}

UFH and LMWH both have been shown to be the best anticoagulant agents for COVID-19 patients. Besides its anticoagulant role, heparin also has the following 2 advantages: an ability to bind to viral proteins and a participation in the downregulation of pro-inflammatory cytokines [31,32] which show high levels in the COVID-19 infected patients [21]. Heparin appears to have a direct antiviral activity by binding to the viral protein $\mathrm{S}$ (spike) and prevents the virus from fusing with the angiotensinconverting enzyme 2 receptor (ACE2) needed for intracellular penetration. Thus heparin behaves as a competitive inhibitor $[14,33]$. The ACE2 receptor is expressed both within the lungs and at the level of the cardiovascular system and it has a critical role in the cardioprotective function of the renin-angiotensin-aldosterone system [34]. The genome of the COVID-19 virus has been identified in post-mortem autopsy of cardiac anatomical samples, suggesting a prompt viral infiltration of myocardial tissue during the infection [35]. Also, in these samples it was observed a marked decrease in the expression of ACE2 receptors simultaneously with an increase in the number of inflammatory cells and the level of fibrosis $[9,36,37]$.

Given the fact that the ACE2 inhibitors represent an important therapeutic line for heart disease with proven beneficial effects and, at the same time, given the limitations of knowing the interactions of the virus with these drugs, it is recommended to continue the treatment with ACE2 inhibitors in patients with clinical indications [20]. ACE2 should be consider "off-label" use to treat those infected with COVID-19 to evaluate the effectiveness of drugs that competitively block the ACE 2 receptor and make it more difficult for coronavirus to enter cells [38]. Reduction of the inflammatory response by heparin in COVID-19 infected patients is achieved by inhibiting neutrophil activation, thus preventing both the expression of inflammatory mediators in the vascular endothelium and the proliferation of vascular smooth muscle cells 
[39]. Adam Torbicki's editorial discusses how the inflammatory response is a major determinant of pulmonary thromboembolism in the COVID-19 infection [40].

According to the algorithm established by the European Society of Cardiology in the management of COVID-19 coagulopathy, the criteria for increased risk of thromboembolism includes: dyspnea, Oxygen (02) saturation less than $90 \%$, respiratory rate greater then 24 breaths/min, high level of C-reactive protein, increased values of D-dimers, fibrinogen and platelets $[4,41]$. The International Society of Thrombosis and Hemostasis and the American Society of Hematology recommend that all patients hospitalized for the COVID-19 infection should receive LMWH thromboprophylaxis $[17,42]$.

Tang et al. showed through a retrospective analysis of 449 patients infected with COVID-19, that the administration of LMWH to 99 of them was correlated with a reduction in mortality. The patients whose levels of D-dimers were more than 6 times the upper limit of the normal had the same benefit. This report highlights the benefit of starting the anticoagulant treatment in COVID-19 infected patients with a score of sepsis-induced coagulopathy $\geq 4$ or with a level of D-dimers $>3 \mu \mathrm{g} / \mathrm{ml}$ [43]. These results are reinforced by another study that was conducted in 17 hospitals in Spain led by Ayerbe et al. which also found that heparin administration was associated with a lower mortality in patients with COVID-19 infection [44].

\section{The impact of chronic anticoagulant treatment in COVID-19 infected patients}

For those patients on chronic oral anticoagulant treatment for other pathologies, switching to LMWH or UFH during COVID-19 infection is recommended, due to the anti-inflammatory and antiviral properties of heparin. Also, due to the fact that p-glycoprotein and cytochrome P450 pathways metabolize direct oral anticoagulants (DOACs) and vitamin K antagonists (VKA), they can cause drug interactions with the antiviral agents [18]. Tesa et al. showed that the plasma levels of DOACs during the treatment of COVID-19 infected patients are 6 times higher during hospitalization compared to the period before hospitalization, probably due to drug-drug interactions. Unlike the oral anticoagulants, the parenteral anticoagulants do not show major drug interactions with antiviral drugs [45].

The thrombotic vascular processes complicate the severe SARS-COV2 infection. The role of chronic anticoagulant therapy before COVID-19 infection and its impact in decreasing the thrombotic risk has not been systematically studied yet. Therefore, Lachant et al. suggested in a retrospective study that the chronic anticoagulant treatment received for at least 1 month before the COVID-19 infection may protect against new thrombotic events and may decrease the severity of the disease. In this study, $61 \%$ of the patients did not require hospitalization. Although these patients were at increased risk of developing new thrombosis, they did not experience any new thrombotic events. Also, the anticoagulant treatment did not cause any unexpected bleeding, nor was there an increased rate of bleeding. Another finding was that for hospitalized patients the median level of D-dimers was lower when compared to other cohorts of hospitalized patients reported in the literature [46]. Middeldrop et al. followed 198 patients hospitalized for COVID-19, $20 \%$ of whom were diagnosed with venous thromboembolism in the first 7 days of hospitalization, although they were given anticoagulant therapy at prophylactic doses. For them, the cumulative incidence of venous thromboembolism at 7, 14 and 21 days was of $16 \%, 33 \%$, respectively $42 \%$. They mentioned that no thrombotic events were found in hospitalized patients who were already using chronic anticoagulant therapy [47]. Consistent with the above-mentioned results, Possy et al. showed that out of the 22 patients with severe acute respiratory syndrome coronavirus 2 (SARS-COV2) whose evolution was complicated by pulmonary thromboembolism, only 2 of them were on chronic anticoagulant therapy [48]. In addition to this data, in the report by Klok et al. conducted on 184 patients, it was observed that the incidence of pulmonary thromboembolism was lower in chronic anticoagulated patients [49]. Similarly, Fauvel et al. included in a multicenter study 1240 patients hospitalized consecutively for COVID-19 infection, afterward they observed that the inpatients that were treated with anticoagulant agents previously to the infection had a lower rate of pulmonary thromboembolism. Of the patients included in the study, 103 (8.3\%) developed pulmonary thromboembolism. 136 patients $(11 \%)$ took chronic anticoagulant medication with heparin, VKA or DOACs, and only 5 of them (4.9\%) developed pulmonary thromboembolism [1].

The American College of Cardiology considers it reasonable to extend the period of thromboembolic prophylaxis after discharge for the high-risk patients (reduced mobility, increased level of D-dimers at discharge, associated comorbidities) [3]. For the outpatients it is recommended to use bleeding scores along with risk scores for venous thromboembolism following by treatment with apixaban $2.5 \mathrm{mg}$ twice daily or rivaroxaban $10 \mathrm{mg}$ daily for 2 weeks [18]. For those conditions with a chronic need for anticoagulation medication, the current crisis brings multiple arguments in favor of DOACs for patients without contraindications. For those patients who took VKA drugs for a long time, this represents an opportunity to change the treatment to DOACs. Hermans etal. recommended that the point-of-care devices should be implemented for measuring the International normalized ratio of prothrombin time (INR) value for patients who must remain on VKA drugs (such as advanced chronic kidney disease, mechanical valve prostheses and antiphospholipid syndrome) [50]. The importance of this fact is underlined by Speed 
et al. who observed that the coumarin overdose is more common in quarantined patients when compared to the period when people could easily go to the medical analysis laboratory.

Due to this current situation, a communication system with three important components has been developed:

i. a structured teleconsultation ('Tele');

ii. an app-based on-demand heart rate and rhythm monitoring infrastructure ('Check'); and

iii. comprehensive AF management ('AF') (TeleCheck-AF) to speak easily with patients who are known with atrial fibrillation to transmit the INR value to the medical staff. It is a recently implemented program so, it has not been possible to assess its effectiveness in controlling the INR value so far.

\section{Conclusions}

All the studies have shown the benefit of LMWH in patients with thromboembolic risk factors and hospitalized for COVID-19 infection. Heparin has other favorable effects besides the anticoagulant one: direct antiviral activity and anti-inflammatory effect. The risk of bleeding should be assessed according to each patient.

D-dimers can be used as an important tool in the clinical trial of each case, having the following advantages: predictive value of identifying thrombosis and, at high level significant correlation with the infection severity and with the progression to acute respiratory distress syndrome coronavirus 2 and also with mortality. The patients that are already receiving chronic anticoagulant therapy for other pathologies have a better outcome of the COVID-19 infection and have a lower rate of adverse events. During the treatment of the infection, it is indicated to change the oral anticoagulant medication with the parenteral one. This crisis has mobilized the medical system for the development of telemedicine for a good access to the medical services to monitor patients evolution.

\section{Author Contributions}

Conceptualization, EB and LAV methodology, LAV validation, LD, MAM, LMR, and LGM; formal analysis, LAV; data curation, LAV; writing-original draft preparation, LAV; writing-review and editing, EB, LGM and AC; visualization, LD, MAM and LMR; supervision, EB, LD, MAM and LMR project administration, EB All authors have read and agreed to the published version of the manuscript.

\section{Funding}

This research received no external funding.

\section{Acknowledgments}

None.

\section{Conflicts of Interest}

The authors declare no conflict of interest.

\section{References}

1. Fauvel C, Weizman 0, Trimaille A, Mika D, Pommier T, et al. (2020) Pulmonary embolism in COVID-19 patients: a French multicentre cohort study. Eur Heart J 41(32): 3058-3068.

2. Al-Ani F, Chehade S, Lazo-Langner A (2020) Thrombosis risk associated with COVID-19 infection. A scoping review. Thromb Res 192: 152160.

3. Becker RC (2020) COVID-19 update: Covid-19-associated coagulopathy. J Thromb Thrombolysis 50: 54-67.

4. Bassam Atallah, Saad I Mallah, Wael AlMahmeed (2020) Anticoagulation in COVID-19. European Heart Journal Cardiovascular Pharmacotherapy 6(4): 260-261.

5. Manne BK, Denorme F, Middleton EA, Portier I, Rowley JW, et al. (2020) Platelet gene expression and function in patients with COVID-19. Blood 136(11): 1317-1329.

6. Bobescu E, Covaciu A, Rus H, Radoi M, Badea M, el al. (2019) Correlation of Cardiovascular Risk Factors and Biomarkers with Platelet Reactivity in Coronary Artery Disease. Am J Ther 26(5): e563-e569.

7. Bobescu E, Covaciu A, Rus H, Rogozea LM, Badea M, et al. (2020) Low Response to Clopidogrel in Coronary Artery Disease. Am J Ther 27(2): e133-e141.

8. Hottz ED, Azevedo-Quintanilha IG, Palhinha L, Teixeira L, Barreto EA, et al. (2020) Platelet activation and platelet-monocyte aggregate formation trigger tissue factor expression in patients with severe COVID-19. Blood 136(11): 1330-1341.

9. Nuche J, Segura de la Cal T, Jiménez López Guarch C, López-Medrano F, Pérez-Olivares Delgado C, et al. (2020) Effect of Coronavirus Disease 2019 in Pulmonary Circulation. The Particular Scenario of Precapillary Pulmonary Hypertension. Diagnostics 10(8): 548.

10. Giannis D, Ziogas IA, Gianni P (2020) Coagulation disorders in coronavirus infected patients: COVID-19, SARS-CoV-1, MERS-CoV and lessons from the past. J Clin Virol 127: 104362.

11. Lüscher TF (2020) Understanding COVID-19: in the end it is the endothelium — what else?. European Heart Journal 41(32): 3023-3027.

12. Steffel J, Luscher TF, Tanner FC (2006) Tissue factor in cardiovascular diseases: molecular mechanisms and clinical implications. Circulation 113(5): 722-731.

13. Betoule A, Martinet C, Gasperini G, Muller P, Foucher S, et al. (2020) Diagnosis of venous and arterial thromboembolic events in COVID-19 virus-infected patients. J Thromb Thrombolysis 50(2): 302-304.

14. Nauka PC, Oran E, Chekuri S (2020) Deep venous thrombosis in a noncritically ill patient with novel COVID-19 infection. Thromb Res 192: 2728.

15. Zamboni P (2020) COVID-19 as a Vascular Disease: Lesson Learned from Imaging and Blood Biomarkers. Diagnostics 10(7): 440.

16. Marchandot B, Sattler L, Jesel L, Kensuke Matsushita, Valerie SchiniKerth, et al. (2020) COVID-19 Related Coagulopathy: A Distinct Entity? J Clin Med 9(6): 1651.

17. Smeda M, Chlopicki S (2020) Endothelial barrier integrity in COVID19-dependent hyperinflammation: does the protective facet of platelet function matter? Cardiovascular Research 116(10): e118-e121.

18. Gavioli E, Sikorska G, Man A, Rana J, Vider E (2020) Current Perspectives of Anticoagulation in Patients With COVID-19. Journal of Cardiovascular Pharmacology 76(2): 146-150.

19. Ky B, Mann DL (2020) CoVID-19 Clinical Trials: A Primer for the Cardiovascular and Cardio-Oncology Communities. JACC Basic Transl Sci 5(5): 501-517. 
20. Porfidia A, Pola R (2020) Venous Thromboembolism and Heparin Use in COVID-19 Patients: Juggling between Pragmatic Choices, Suggestions of Medical Societies. J Thromb Thrombolysis 50(1): 68-71.

19. Inciardi RM, Adamo M, Lupi L, Cani DS, Di Pasquale M, et al. (2020) Characteristics and outcomes of patients hospitalized for COVID-19 and cardiac disease in Northern Italy. Eur Heart J 41(19): 1821-1829.

20. Zhou F, Yu T, Du R, Fan GT, Liu Y, et al. (2020) Clinical course and risk factors for mortality of adult inpatients with COVID-19 in Wuhan, China: a retrospective cohort study. Lancet 395(10229): 1054-1062.

21. Kang Y, Chen T, Mui D, Ferrari V, Jagasia D, et al. (2020) Cardiovascular manifestations and treatment considerations in covid-19. Heart 106(15).

22. Mao L, Jin H, Wang M, Hu Y, Chen S, et al. (2020) Neurologic manifestations of hospitalized patients with coronavirus disease 2019 in Wuhan, China. JAMA Neurol 77(6): 683-690.

23. Chachques JC, Mazzini L, Mitrecic D, Mitrecic D, Hellmich C et al. (2020) Covid-19 Pandemic: Cardiovascular and Neurologic Impact, Early Signs and Symptoms. Am J Biomed Sci \& Res 11(1): 51-63.

24. Sanchis-Gomar F, Perez-Quilis C, Lavie C (2020) Should atrial fibrillation be considered a cardiovascular risk factor for a worse prognosis in COVID-19 patients? European Heart Journal 41(32): 3092-3093.

25. Holt A, Gislason GH, Schou M, Zareini B, Biering-Sørensen T, et al. (2020) New-onset atrial fibrillation: incidence, characteristics, and related events following a national COVID-19 lockdown of 5.6 million people. Eur Heart J. 41(32): 3072-3079.

26. Dherange P, Lang J, Qian P, Oberfeld B, Sauer WH, et al. (2020) Arrhythmias and COVID-19: A review. JACC: Clinical Electrophysiology 6(9): 1193-1204.

27. Lazzerini PE, Laghi-Pasini F, Acampa M, Srivastava U, Bertolozzini J, et al. (2019) Systemic inflammation rapidly induces reversible atrial electrical remodeling: the role of interleukin-6-mediated changes in connexin expression. J Am Heart Assoc 8(16): e011006.

28. Llitjos JF, Leclerc M, Chochois C, Monsallier JM, Ramakers M, et al. (2020) High incidence of venous thromboembolic events in anticoagulated severe COVID-19 patients. J Thromb Haemost 18(7): 1743-1746.

29. Ranucci M, Ballotta A, Di Dedda U, Bayshnikova E, Dei Poli M, et al. (2020) The procoagulant pattern of patients with COVID-19 acute respiratory distress syndrome. J Thromb Haemost 18(7): 1747-1751.

30. Mummery RS, Rider CC (2000) Characterization of the heparin-binding properties of IL-6. J Immunol 165(10): 5671-5679.

31. Hippensteel JA, LaRiviere WB, Colbert JF, Langouët-Astrié CJ, Schmidt EP (2020) Heparin as a therapy for COVID-19: current evidence and future possibilities. Am J Physiol Lung Cell Mol Physiol 319(2): L211-L217.

32. Ganatra S, Hammond SP, Nohria A (2020) The Novel Coronavirus Disease (COVID-19) Threat for Patients With Cardiovascular Disease and Cancer. JACC CardioOncol 2(2): 350-355.

33. Oudit GY, Kassiri Z, Jiang C, Liu PP, Poutanen SM, et al. (2009) SARS- coronavirus modulation of myocardial ACE2 expression and inflammation in patients with SARS. Eur J Clin Invest 39(7): 618-625.

34. Oudit GY, Pfeffer MA (2020) Plasma angiotensin-converting enzyme 2: novel biomarker in heart failure with implications for COVID-19. European Heart Journal 41(19): 1818-1820.
35. Schreiber B, Patel A, Verma A (2020) Shedding Light on COVID-19. American Journal of Therapeutics.

36. Koprowski EJ (2020) Are Off-Label Prescriptions of ACE Inhibitors a Promising Therapy for COVID-19? AJBSR 9(6): 447.

37. Poterucha TJ, Libby P, Goldhaber SZ (2017) More than an anticoagulant: do heparins have direct anti-inflammatory effects? Thromb Haemost 117(3): 437-444.

38. Torbicki A (2020) COVID-19 and pulmonary embolism: an unwanted alliance. Eur Heart J 41(32): 3069-3071.

39. Mazzaccaro D, Giacomazzi F, Giannetta M, Varriale A, Scaramuzzo R, et al. (2020) Non-Overt Coagulopathy in Non-ICU Patients with Mild to Moderate COVID-19 Pneumonia. J. Clin. Med 9(6): 1781.

40. Ambrosino P, Di Minno A, Maniscalco M, Di Minno (2020) COVID-19 and venous thromboembolism: current insights and prophylactic strategies, Annals of Medicine, 52(6): 239-242.

41. Tang N, Bai H, Chen X, Gong J, Li D, et al. (2020) Anticoagulant treatment is associated with decreased mortality in severe coronavirus disease 2019 patients with coagulopathy. J Thromb Haemost 18(5): 1519-1520.

42. Ayerbe L, Risco C, Ayis S (2020) The association between treatment with heparin and survival in patients with Covid-19. J Thromb Thrombolysis 50(2): 298-301.

43. Testa S, Prandoni P, Paoletti, Morandini R, et al. (2020) Direct oral anticoagulant plasma levels' striking increase in severe COVID-19 respiratory syndrome patients treated with antiviral agents. The Cremona experiences. J Thromb Haemost 18(6): 1320-1323.

44. Lachant DJ, Lachant NA, Kouides P, Rappaport S, Prasad P, et al. (2020) Chronic therapeutic anticoagulation is associated with decreased thrombotic complications in SARS-CoV-2 infection. J Thromb Haemost.

45. Middeldorp S, Coppens M, van Haaps TF, Foppen M, Vlaar AP, et al. (2020) Incidence of venous thromboembolism in hospitalized patients with COVID-19. J Thromb Haemost 18(8): 1995-2002.

46. Poissy J, Goutay J, Caplan M, Parmentier E, Duburcq T, et al. (2020) Pulmonary embolism in COVID- 19 patients: awareness of an increased prevalence. Circulation 142: 184-186.

47. Klok FA, Kruip MJHA, van der Meer NJM, Arbouse MS, Gommerset D, et al. (2020) Confirmation of the high cumulative incidence of thrombotic complications in critically ill ICU patients with COVID-19: An updated analysis. Thromb Res 3848(20): 148-150.

48. Hermans C, Lambert C (2020) Impact of the COVID-19 pandemic on therapeutic choices in thrombosis-hemostasis. J Thromb Haemost 18(7): 1794-1795.

49. Speed V, Patel RK, Byrne, Roberts LN, Arya R, et al. (2020) A perfect storm: Root cause analysis of supra-therapeutic anticoagulation with vitamin K antagonists during the COVID-19 pandemic. Thromb Res 192: 73-74.

50. Linz D, Pluymaekers NAHA, Hendriks JM (2020) TeleCheck-AF for COVID-19. Eur Heart J 41(21): 1954-1955. 